\title{
La frontière et au-delà. Une enquête ethnographique sur le narcotrafic à Ciudad Juárez (Mexique) et El Paso (Etats-Unis)
}

The border and beyond: an ethnographic investigation on drug trafficking in Ciudad Juarez and El Paso

La frontera y más allá. Investigación etnográfica sobre el narcotráfico en Ciudad Juárez y El Paso

\section{Sabine GUEZ}

\section{(2) OpenEdition}

\section{Journals}

Édition électronique

URL : http://journals.openedition.org/conflits/17363

DOI : $10.4000 /$ conflits. 17363

ISSN : $1777-5345$

Éditeur :

CCLS - Centre d'études sur les conflits lilberté et sécurité, L'Harmattan

Édition imprimée

Date de publication : 20 décembre 2008

Pagination : 13-29

ISBN : 1157-966 X

ISSN : 1157-996X

\section{Référence électronique}

Sabine GUEZ, « La frontière et au-delà. Une enquête ethnographique sur le narcotrafic à Ciudad Juárez (Mexique) et El Paso (Etats-Unis) », Cultures \& Conflits [En ligne], 72 I hiver 2008, mis en ligne le 19 mai 2009, consulté le 30 mars 2021. URL : http://journals.openedition.org/conflits/17363 ; DOI : https:// doi.org/10.4000/conflits. 17363 


\section{La frontière et au-delà. Une enquête ethnographique sur le narcotrafic à Ciudad Juárez (Mexique) et El Paso (Etats-Unis) 1}

\section{Sabine GUEZ}

Sabine Guez est doctorante en anthropologie sociale à l'Ecole des Hautes Etudes en sciences sociales et membre de l'IRIS (Institut de recherche interdisciplinaire sur les enjeux sociaux). Elle a récemment publié : $A$ la frontière $d u$ légal et de l'illégal, travail et narcotrafic à Ciudad Juárez (Mexique) et El Paso (Etats-Unis) ", Problèmes d'Amérique latine, n66-67, automne-hiver 2007, pp. 9-20.

\section{Le contexte}

$\mathbf{R}$ eplaçons dans leur contexte les deux histoires / carrières individuelles autour desquelles s'articule cet article : l'essor du trafic de drogue dans le Nord mexicain depuis les années 1980.

Plus de 530 tonnes de cocaïne en provenance d'Amérique du Sud parviendraient aux Etats-Unis chaque année - dont $90 \%$ transiteraient par le Mexique ${ }^{2}$. Ciudad Juárez, côté mexicain, et El Paso, côté états-unien, forment

1. Je remercie mes interlocuteurs à la frontière, mes partenaires dans ce travail, qui m'ont appris ce que je sais de Ciudad Juárez et d'El Paso. Mes remerciements vont aussi aux évaluateurs anonymes de Cultures $\mathcal{E}$ Conflits, pour leur lecture attentive de ce texte et leurs précieux conseils.

2. Estimation de David Johnson, Assistant Secretary for International Narcotics and Law Enforcement Affairs, introduction au rapport annuel International Narcotics Control Strategy Report, 29 février 2008 (http://www.state.gov/p/inl/rls/rm/101564.htm). La quantité de cocaïne sud-américaine arrivant à destination se situerait dans une fourchette comprise entre 530 et 710 tonnes en 2006, lit-on dans ce rapport (p. 18). Ces chiffres fournissent un ordre de grandeur, mais les estimations sur le trafic, en raison même de sa nature secrète, sont sujettes à caution et il n'y a pas deux sources officielles qui s'accordent sur les montants. Les statistiques concernant le volume des saisies - 96,7 tonnes de cocaïne de janvier à décembre 2007; 69,8 tonnes en 2006, selon la Drug Enforcement Administration (DEA) 
une conurbation de 2,5 millions d'habitants, encerclée par le désert et sise à mi-chemin des 3200 kilomètres de frontière entre les deux nations. Cette situation géographique, couplée à l'essor régional de l'infrastructure des transports notamment autoroutiers, en fait une frontière pivot sur la route transaméricaine de la cocaïne, de la marijuana, de l'héroïne et de la méthamphétamine. L'économie de la drogue y occupe aujourd'hui directement des dizaines de milliers de personnes, et elle y intéresse un nombre incalculable de participants à des "activités de renfort ${ }^{3}$ ", en raison même du poids de ce trafic et de la circulation spectaculaire de revenus d'origine illégale. Le narcotrafic, comme activité collective a happé des membres de toutes les classes sociales à partir des années 1980. Jusque-là, le Mexique était principalement un pays de culture de cannabis et de pavot, et d'exportation de marijuana et d'héroïne. Une nouvelle période s'est ouverte avec les années 1980.

Sous le mandat de Ronald Reagan, la fermeture de la Floride en tant que point d'entrée de la cocaïne amène les trafiquants colombiens à renforcer leurs alliances avec leurs homologues mexicains. Conséquence bien involontaire de la politique fédérale états-unienne en matière de répression des flux de stupéfiants ${ }^{4}$, le transit - désormais de masse - de la cocaïne par le Mexique produit un changement d'échelle économique du trafic de drogue dans ce pays ${ }^{5}$. Des fonctionnaires de tous les niveaux et des profesionistas (diplômés) de secteurs économiques variés ne résistent pas aux possibilités d'enrichissement qui en résultent. En bas de l'échelle sociale, dans les secteurs ruraux mais aussi dans des villes comme Juárez, le trafic ou la petite distribution pallient parfois les effets déstructurants des crises financières en série (1982, 1994), de l'intégra-

(http://www.usdoj.gov/dea/statistics.html\#seizures) - restent partielles, selon David Monnette, chargé de leur diffusion pour la DEA, puisqu'elles n'incluent pas les saisies effectuées par les agences au niveau local et des Etats (entretien, juin 2006). Bien qu'elles n'évitent pas ce biais, les estimations du Federal-Wide Drug Seizure System, qui regroupe les données sur les saisies apportées par la DEA, le FBI, le Customs Service et la Border Patrol, restent les plus fiables: 150 tonnes de cocaïne saisies en 2006, dont 95 en haute mer et 55 à terre (National Drug Threat Assessment 2008, http://www.usdoj.gov/ndic/topics/ndtas.htm\#Top, p. 78). Mes interlocuteurs au sein des agences fédérales et locales semblent d'accord sur un point : les quantités saisies représenteraient au mieux le dixième des quantités qui franchissent la frontière.

3. Suivant la terminologie de H. S. Becker au sujet des Mondes de l'art, Paris, Flammarion, 1988 (1 ${ }^{\text {re }}$ éd. 1982), les "activités de renfort» sont des activités indirectement liées au narcotrafic - par exemple, défendre un trafiquant devant la justice. Ou encore, comme on le verra dans les deux cas développés ci-après : faciliter le travail de trafiquants depuis l'intérieur du gouvernement; venir en aide à l'un d'eux qui est votre ami.

4. Toro M.C., Mexico's “War” on Drugs. Causes and Consequences, Boulder, Colorado, Lynne Rienner Publishers, Inc., 1995, p. 31.

5. Sur le changement d'échelle économique du trafic de drogue au Mexique à partir des années 1980, voir Astorga L., El siglo de las drogas, México, Plaza \& Janés, 2005 (1 ${ }^{\mathrm{re}}$ éd. 1996), p. 123. Les données sur les saisies en territoire mexicain incluent la cocaïne à partir du début des années 1960. Son transit par le Mexique en plus grande quantité date des années 1970, mais le trafic de la cocaïne est alors sans commune mesure avec ce qu'il deviendra à partir de la décennie suivante : entre 1970 et 1976, une seule tonne de cocaïne est saisie, voir ibid., p. 108 et p. 121, et Astorga L., "Cocaine in Mexico: a Prelude to 'Los Narcos'”, in Gootenberg, P., Cocaine. Global Histories, Routledge, London, 1999. 
tion croissante des économies asymétriques des Etats-Unis et du Mexique et de l'ouverture débridée de l'agriculture nationale à la concurrence étrangère. Dans les années 1980 et 1990, de bas en haut de l'échelle sociale, les activités liées au trafic sont rationalisées et les normes se relâchent. Les frontières de l'espace moral du possible et du légitime bougent ${ }^{6}$.

\section{Des usages de la frontière}

Le narcotrafic à la frontière gagne à être étudié en variant les focales d'observation ${ }^{7}$. Des décisions prises à Washington ou à Mexico produisent localement des effets pervers; se pencher sur la genèse de ces décisions éclaire notamment "la popularité de la frontière comme scène politique 8 ", pour reprendre les termes de Peter Andreas. Ce politologue décrit le jeu des postures goffmanien auquel se livrent des acteurs politiques en exercice loin de la frontière : ils se servent de celle-ci pour figurer, à l'adresse d'un électorat national ou du gouvernement voisin, l'image que l'Etat (tel un seul homme) veut projeter de son inflexibilité ${ }^{9}$. Au même moment, à Ciudad Juárez et à El Paso, des histoires individuelles se jouent. Parce qu'ils vivent dans un monde binational, plus riche que la somme de ses parties, les acteurs frontaliers sont sensibles à ces jeux d'échelle, attentifs aux réorientations des politiques fédérales «de l'autre côté » car elles retentissent sur leurs pratiques (par exemple, par l'allongement de la durée d'attente au poste-frontière étatsunien). Ils sont exposés à des narrations nationales discordantes, ils les écoutent mais d'une oreille pragmatique ; quel que soit leur degré d'identification et d'affinité avec ces discours, ils apprennent à changer d'babitus et à calquer leurs manières de faire sur celles qui ont cours une fois franchie la ligne de démarcation des territoires. Des habitants du monde frontalier, résidents ou de passage, deviennent maîtres dans l'art de se réapproprier les politiques de contrôle et de répression des flux illégaux. En l'espèce, d'aucuns saisissent des occasions et/ou se retrouvent à participer au trafic, y compris à sa marge, situations créées par la demande considérable de drogues aux Etats-Unis, et désormais aussi au Mexique. A leur échelle également, mes interlocuteurs instrumentalisent la frontière, chacun à leur manière.

Deux d'entre eux ont joué pour moi le rôle de "passeurs » lors de mon huitième et plus long séjour à Ciudad Juárez et à El Paso (de septembre 2005 à

6. Sur l'essor de l'économie de la drogue au Mexique comme activité collective et sur ce processus de relâchement normatif, voir Guez S., "A la frontière du légal et de l'illégal, travail... ", op. cit.

7. Suivant les travaux des micro-historiens, voir Revel J., «L'histoire au ras du sol » in Levi G., Le Pouvoir an village. Histoire d'un exorciste dans le Piémont du XVII siècle, Préface, Paris, Gallimard, 1989, pp. I-XXXIII.

8. Andreas P., Border Games. Policing the US-Mexico Divide, Ithaca, NY, Cornell University Press, 2000, pp. 9-11.

9. Goffman E., «La présentation de soi », La Mise en scène de la vie quotidienne, t. 1, Paris, Editions de Minuit, 1973. 
août 2006), d'éclaireurs de l'histoire sociologique contemporaine de l'essor de l'économie de la drogue, dans cette région au Nord du Mexique et au SudOuest des Etats-Unis. Cet homme et cette femme, issus de la classe moyenne supérieure, ne se connaissent pas. Bien qu'ils se tiennent à la lisière du trafic de drogue, ils sont situés au centre d'un réseau d'interconnaissances dans ce "bas monde ", comme le nomment parfois les Juarenses eux-mêmes impliqués. L'un et l'autre en ont acquis, de par leur profession et/ou leurs amitiés, un savoir personnel et pratique. Souvent, leurs propos réflexifs sur un « avant-ailleurs » du narcotrafic qu'ils avaient connu m'enjoignaient d'élargir le regard sur la frontière à son au-delà. J'ai pu le faire grâce à leur accès à d'autres acteurs. Je tenterai, dans cet article, de montrer l'extension de la frontière bien au-delà des villes de Juárez et El Paso, à la lumière des trajectoires évoquées en pointillé de personnes situées à la jonction de ce commerce illégal : deux personnes rencontrées grâce à mes deux « guides » ou interlocuteurs privilégiés. Des fragments d'entretien, l'ébauche de leurs portraits seront une manière d'incarner - en deux zooms successifs, sur deux mondes (parmi d'autres) et deux moments dans l'histoire du narcotrafic (les années 1980 et la période actuelle) - des complexes d'usages 10 de la frontière et l'articulation de ses rapports avec l'intérieur mexicain et états-unien. J'essaye donc de penser cette frontière dans ses interactions avec les territoires, les personnes, les projets qu'elle relie et met en réseau. Part belle sera faite ici aux propos des acteurs et à leurs actions resituées dans leur contexte - un mode d'exposition en écho à la forte individualisation des relations sociales sur le «terrain ", sans laquelle il n'y a pas d'enquête ethnographique sur ce trafic. Enquête dont il faut commencer par dire quelques mots des conditions dans lesquelles elle a été réalisée.

\section{A objet délicat, conditions d'enquête malaisées}

Ce matériau ethnographique est le produit de conditions d'enquête à chaque fois différentes, mais rarement optimales. Si j’ai pu partager le quotidien de plusieurs de mes interlocuteurs, avec d'autres, les rencontres furent peu nombreuses, brèves et espacées. Ainsi avec Ricardo, un trafiquant dont il sera question plus loin dans cet article, résidant à Chihuahua, capitale de l'Etat du même nom à l'extrême Nord duquel la ville de Juárez est située. Je ne le voyais que lors de ses haltes à El Paso, avant qu'il ne reprenne la route pour l'intérieur des Etats-Unis où il était attendu pour «affaires».

S'agissant de Don Silvio, il n'y eut qu'une seule rencontre, quelques jours avant mon retour en France. Il avait officié durant plus de trente ans au sein du gouvernement à des postes d'observation clés du trafic et de ses ramifica-

10. On peut aussi parler de grammaires des usages sociaux, politiques, économiques de la frontière : ici esquissées, celle des trafiquants et de leurs facilitateurs au sein de l'Etat, que le portrait de Don Silvio fait apparaître, et celle d'un petit trafiquant indépendant, Ricardo. 
tions politiques, économiques et sociales. L'homme qui fut mon «passeur »- appelons-le Roberto - avait attiré son ami Silvio dans un grand restaurant de Ciudad Juárez en l'y invitant à déjeuner en sa compagnie et celle $\mathrm{d}$ ' « une jeune femme française ». Roberto est un homme grand, aux traits fins et aux manières élégantes d'un autre temps, un homme de culture littéraire et politique, homme de réseau, discret, très bien introduit dans les cercles du pouvoir, au niveau local et fédéral. Il a près de 70 ans, un esprit pince-sans-rire et vif. Ce n'est qu'une fois à table qu'il expliqua à Don Silvio de quoi il retournait, lui parlant de «notre » travail : Roberto - un avocat de renom jouissant de toute l'estime de Don Silvio - et moi, «travaillions » sur les rapports entre narcotrafic et Etat. Don Silvio hésita, sur ses gardes. Roberto l'avait piégé : lui eût-il dit à l'avance le pourquoi de cette rencontre, Don Silvio aurait répondu «Tu es fou ! »... ou alors il n'aurait rien répondu du tout et on l'attendrait toujours. Rassuré par les regards de Roberto, sa confiance à mon endroit, Don Silvio finit par entrer dans le vif du sujet, non sans d'abord maintenir un certain flou, le temps de tester mes connaissances. Puis Roberto s'éclipsa une fois Don Silvio lancé, et la conversation se prolongea dans les bureaux de l'ancien fonctionnaire, longtemps après la tombée du jour.

Cependant, sa paranoïa resurgissait, resurgirait. Ainsi, tandis qu'on arrivait à bord de sa voiture à hauteur de la maison abritant son cabinet il concluait sur un point d'histoire quand, soudain, il dit :

«-C’est si intéressant... mais tellement dangereux ! Tu n’es pas de la CIA ?

- [Surprise par sa question abrupte] De la quoi ?

- De la CIA ? Tu n'es pas de la CIA ? Tu ne travailles pas pour la CIA ? Je te pose la question. Dis-moi la vérité ! Dis !

- Mais je suis anthropologue!

- Tu es de la CIA, c'est ça ? Regarde-moi dans les yeux. Oui ou non? Yes or no ? 11 »

Suspicion et sommations ${ }^{12}$, illustration de la paranoïa ordinaire et scène banale de jeu de pouvoir... Don Silvio voulait aussi et surtout voir si j'avais peur et rire un peu. Mais il se souvint sans doute que notre ami Roberto m'avait confiée à lui car il remit à plus tard ses questions et il poursuivit son récit.

11. Toutes les traductions vers le français (de l'espagnol et de l'anglais) sont de Sabine Guez.

12 . Je lui dis souhaiter qu'il fasse jouer son réseau d'informateurs en ville. " En 72 beures, on sait tout à Ciudad Juárez », assurait-il. A l'époque où il travaillait pour les services secrets, il « disposai $[\mathrm{t}]$ de 200-300 personnes - un réseau de serveurs, d'employés de banque, de compères, d'associés, de prostituées, d'enfants... ». Je l'y incitai à plusieurs reprises tout en craignant que, s'il venait à la découvrir, l'origine de ma bourse ne nourrisse ses doutes au lieu de les apaiser. (En effet, l'enquête sur laquelle s'appuie cette recherche a été réalisée à l'aide d'une bourse de la Fondation Fulbright et durant l'année d'enquête de terrain, j'étais affiliée à l'université du Texas à El Paso). 


\section{Ciudad Juárez - la carrière de Don Silvio. Au temps d'avant (avant le tournant des années 1980 13)}

Les liens entre narcotrafiquants et agents sociaux représentant l'ordre ou incarnant le pouvoir politique sont anciens, et leur histoire est constamment actualisée. Dernier exemple en date : Saulo Reyes Gamboa, toujours directeur de la police municipale juarense en 2007. Comptable de profession, titulaire d'une maîtrise de droit fiscal, il avait occupé, entre autres fonctions, celle de trésorier dans les administrations municipales depuis 1995. Le 16 janvier 2008, il a été interpellé à El Paso alors qu'il versait à un douanier états-unien (qu'il croyait corrompu) la somme de 15000 dollars - son second paiement en deux jours - pour avoir laissé passer le jour même une cargaison d'environ $500 \mathrm{~kg}$ de marijuana ${ }^{14}$. Le témoignage de Don Silvio, vétéran des services secrets, apporte un éclairage supplémentaire à cette histoire documentée par les sciences sociales ${ }^{15}$.

L'homme se félicite d'être encore en vie. Natif de Ciudad Juárez, il a la cinquantaine nerveuse, le regard rieur, le geste large et professoral. Pendant toutes ses années au gouvernement, il enseignait aussi à l'université et, durant notre entretien, Don Silvio arpente son bureau, réfléchissant et racontant. Il considère la Secretaría de Gobernación ${ }^{16}$, où il est recruté à l'âge de 22 ans et où il a travaillé deux décennies, comme sa «maison... C'est là où je suis né ».

13. Les années 1980 sont un tournant à un autre titre. Pendant la décennie, deux maillons clefs dans la chaine de coopération entre les trafiquants et leurs protecteurs au sein de l'Etat mexicain sautent : en 1985, un agent de la DEA états-unienne en poste au Mexique est assassiné par des trafiquants et des policiers travaillant à leur solde, et la crise bilatérale qui s'ensuit aboutit à la disparition de la Dirección Federal de Seguridad (DFS) et du système de contrôle qui sera décrit ici par Don Silvio ; une autre entité médiatrice, le Partido Revolucionario Institucional, est affaiblie par la montée de l'opposition et, dès 1989, son ascension au pouvoir dans un Etat du Nord. La dissolution de la DFS et l'éclatement de l'ancienne structure du pouvoir priista créent les conditions de possibilité d'une plus grande autonomie des principales organisations de trafiquants vis-à-vis de l'Etat. Voir Astorga L., “The Field of Drug Trafficking in Mexico”, in Geffray et al. (eds.), Globalisation, Drugs and Criminalisation, UNESCO, MOST-drugs Programme, Paris, 2002 http://www.unesco.org/most/globalisation/drugs_1.htm, pp. 65-72.

14. Figueroa L., "Cae por narco », El Diario de El Paso, 18 janvier 2008.

15. Le présent travail prend appui sur les travaux pionniers de socio-histoire du narcotrafic réalisés par le sociologue mexicain Luis Astorga, qui a étudié l'émergence du champ social du trafic de drogue en relation avec le champ politique au Mexique, voir notamment El siglo de las drogas, op. cit.; "The Field of Drug Trafficking in Mexico", op. cit. ; "The Social Construction of the Identity of the Trafficker" in Geffray et al. (eds.), op. cit. Voir aussi Geffray C., "Introduction: Drug Trafficking and the State”, in Geffray et al. (eds.), op. cit. ; Resa Nestares C., El estado como maximizador de rentas del crimen organizado: El caso del tráfico de drogas en México, Barcelone, Instituto Internacional de Gobernabilidad, Documento de Trabajo n ${ }^{\circ} 88$, octobre 2001 ; IMECO (Instituto Mexicano de Estudios de la Criminalidad Organizada), Todo lo que debería saber sobre el crimen organizado en México, México, Océano, 1998 ; Lupsha P.A., "Drug Lords and NarcoCorruption: The Players Change but the Game Continues”, Crime, Law and Social Change, n¹6, 1991, pp. 41-58 ; Pimentel S., "The Nexus of Organized Crime and Politics in Mexico”, Trends in Organized Crime, 1999, vol. 4, n³, pp. 9-27.

16. Les attributions de la Secretaría de Gobernación recouvrent en partie celles du ministère de l'Intérieur français. «Gobernación » coordonne notamment la politique de sécurité nationale 
« Au début, t’imagines aider ton pays. Après... tu deviens frustré, tu prends peur, et tu te demandes : qu'est-ce que je fais ici ? [...] Dans un premier temps, ça me fascine, ensuite je suis gagné par la panique... Parce que tous sont mouillés et ils savent que tu sais. »

Il planche d'abord sur les questions électorales puis entre au service des renseignements. Il est amené à rencontrer et à fréquenter des personnes dont plusieurs, des trafiquants, le prennent en amitié, lui font des confidences, qui le lient. Il se sait en permanence sur le fil. Son supérieur hiérarchique d'alors et son subalterne immédiat ont été assassinés depuis. "Lui ne s'est jamais enrichi. C'est pour ça qu'il est en vie ", dit Roberto.

Les routes des uns et des autres - grands trafiquants et fonctionnaires publics - se sont croisées dès l'enfance et la première scolarisation. En 1960, Ciudad Juárez ne comptait que 250000 habitants contre 1,4 million aujourd'hui. Sur les bancs du seul lycée de l'époque, Don Silvio côtoie des hommes qui deviendront ses collaborateurs et amis, comme Rafael Aguilar Guajardo, délégué régional de la Dirección Federal de Seguridad (DFS) en charge du Nord-Est mexicain. Il est l'un des supérieurs de Don Silvio au moment où celui-ci occupe un poste de responsabilité à la Dirección General de Investigaciones Politicas y Sociales (DGIPS), à Ciudad Juárez. La DFS (1947) et la DGIPS (1967) 17 _ parfois surnommées la «CIA MEX »-sont le noyau du dispositif de renseignement mexicain jusqu'à leur démantèlement dans les années 1980 18. Mais Aguilar est mieux connu pour avoir cofondé ledit «Cartel de Juárez », l'une des principales organisations criminelles nord-américaines, présente dans pas moins de 44 villes des Etats-Unis ${ }^{19}$. Comme lui, d'autres grands trafiquants ont fait leurs armes à la DFS, plusieurs au sein de sa «Brigade blanche 20 ", l'unité de police secrète qui livre la « guerre sale » contre les mouvements de gauche et les guérilleros dans les années 1970. A la DFS, ils garantissent à un titre ou un autre la protection de champs de cannabis et de pavot, de convois de stupéfiants et celle de trafiquants ${ }^{21}$. Ce faisant, ils réalisent qu'ils pourraient

(voir la Loi organique de l'administration publique fédérale, Titre second, chap. II, article 27). Son titulaire est - après le président - l'individu le plus puissant au sein de l'Etat mexicain.

17. La DGIPS faisait de l'analyse stratégique, la Dirección Federal de Seguridad générait l'information.

18. Ces deux agences fédérales fusionnent et deviennent en 1985 la Dirección General de Investigación y Seguridad Nacional (DISEN), renommée Centro de Investigación y Seguridad Nacional (CISEN) quatre ans plus tard.

19. "Cities in which Mexican DTOs Operate within the United States", Situation Report, US Department of Justice, National Drug Intelligence Center, 11 avril 2008.

20. Neuvième brigade de la División de Investigaciones para la Prevención de la Delincuencia (DIPD), la « Brigade blanche » était composée de membres des divers corps de police et de l'armée et spécialisée dans les détentions, la torture, les disparitions et les exécutions, de sa création en juin 1976 pour « anéantir » la Liga Comunista 23 de Septiembre, jusqu’à sa dissolution en janvier 1983. Torres J., « DFS : la misión, aniquilar », Proceso, n¹478, 27 février 2005, p. 20.

21. Le Mexique demeure le premier pourvoyeur de marijuana et de méthamphétamine du voisin $\mathrm{du}$ Nord, en plus d'être son deuxième plus gros fournisseur d'hérö̈ne (bien qu'il produise 
faire aussi bien que ces derniers et gagner mieux que leur quote-part dans les profits du trafic. Forts de leur sentiment d'impunité né de la perpétration des pires crimes politiques, ils décident de se consacrer à temps plein à ce négoce illégal, autrement dit, de passer du public au privé.

Le système DFS/DGIPS est à de nombreux égards révélateur de la perméabilité empirique de la frontière entre légalité et illégalité et de son caractère idéologique ${ }^{22}$. Les hommes et les femmes travaillant pour ces agences gouvernementales agissent dans un entre-deux-monde : entre légalité et illégalité, au nom de l'Etat, sous couvert de (et non pas hors) la loi, et ceci au service d'une activité illicite. Dans cet espace à "l'intersection entre le gouvernement et l'illégalité 23 ", la Secretaría de Gobernación (tutelle des services secrets) n'est ni le dernier ni le seul des cercles concernés par la protection du narcotrafic dans la période qu'a connue Don Silvio (années 1970 et 1980) :

- [Don Silvio] : «Ils se répartissaient le travail, une partie à l'armée, une autre aux fédéraux en personne [le ministère de la Justice] et débarque un troisième larron : "et nous alors ?" [rires de Don Silvio et de Roberto]. A la Secretaría de Gobernación on était bien sûr dans le coup...»

Le travail de la DFS et de la DGIPS est alors distribué en sept zones sur le territoire national. A la DGIPS, Don Silvio est la cheville ouvrière, postée à Ciudad Juárez, d'une stratégie élaborée à Mexico. Il a surnommé sa région du Nord - d'importance cardinale pour les narcotrafiquants et ceux qui les taxent - «le triangle de la mort ». Dans l'extrait d'entretien qui suit, il met en scène des pratiques politiques d'entente avec les trafiquants et il expose les intérêts en conflit d'agents au sein de l'Etat, ici «démasqué » par Don Silvio, selon le terme de Philip Abrams 24.

moins de $3 \%$ du total mondial), https://www.cia.gov/library/publications/the-world-factbook/geos/mx.html\#Issues; International Narcotics Control Strategy Report, Bureau for International Narcotics and Law Enforcement Affairs, February 2008,

http://www.state.gov/p/inl/rls/nrcrpt/2008/; http://www.whitehousedrugpolicy.gov/publications/pdf/heroin2002.pdf

22 . Heyman J. McC., Smart A., "States and Illegal Practices: An Overview”, in Heyman J. McC. (ed.), States and Illegal Practices, Oxford, Berg, 1999, pp. 1-24. Sur l'imbrication des liens entre les chefs du narcotrafic et ceux du renseignement dans les années 1970-1980, voir Shannon E., Desperados, New York, Penguin, 1988.

23. Heyman J. McC., Smart A., op. cit., p. 2.

24. L'Etat est «le masque qui nous empêche de voir [1]es pratiques politiques », écrit Abrams dans un essai qui fit date, voir: "Notes on the Difficulty of Studying the State", Journal of Historical Sociology, n¹, 1988, pp. 58-89. Pour Abrams, l'idée d'Etat « un », réifié, dissimule sa désunion et sa complexité interne et il importe de la déconstruire si l'on veut étudier les pratiques étatiques. 
«- [DS] : Historiquement, le gouvernement mexicain contrôlait, conditionnait, exterminait, faisait disparaitre, ordonnait. [...] Depuis que le narcotrafic existe [...] - parce que le narcotrafic constitue un crime fédéral - le gouvernement fédéral le gérait. Cependant, au fil du temps, de compromis politique en compromis, le gouvernement fédéral a commencé à déléguer le contrôle du narco à certains gouverneurs, tu vois. En trois mots, le gouvernement dirigeait et contrôlait... Non [se corrigeant]. Il contrôlait mais il ne peut pas le diriger, il n'a jamais pu le diriger. Jusqu'à la chute du vieux système [c'est-à-dire jusqu'à l'effondrement du système qu'il décrit] [...] il contrôlait : comment ? Il mettait des limites, il assignait des territoires, il fixait des quotas, il balisait des comportements. Et il exigeait des résultats. OK ? Les capos ont toujours existé... Par l'intermédiaire de leurs avocats, de leurs administrateurs, de leurs comptables, ils approchaient et établissaient le contact avec les gens du pouvoir. [...] Comment un paysan qui ne sait ni lire ni écrire fait pour aborder un gouverneur ou un chef de la police fédérale ? C’est très dur... Mais ses conseillers - ou donneleur le nom que tu voudras - lui servent de courroie de transmission. [...] Donc, le gouvernement acceptait et imposait des quotas :

- "Tu sais quoi ? Je vais te laisser bosser Chihuahua. T'es en charge de Chihuahua et j'en attends 500000 dollars par semaine mais attention... en plus des 500000 dollars par semaine..."

[Il s'interrompt pour me dire :] Camarade, ces quantités sont à titre d'exemple...

$-[\mathrm{SG}]$ : Oui.

- [DS, reprenant, cette fois dans le rôle du capo] : “500 000 dollars pour gérer Ciudad Juárez et la place 25 est à moi. Je gère la place...

- [Dans le rôle du fonctionnaire du gouvernement fédéral ou de l'Etat de Chihuahua] “... Pas si vite. En plus des 500000 dollars par mois, je ne veux pas de morts, je ne veux pas d'assassinats, je ne veux pas de violence, je ne veux pas que tu t'exhibes, je ne veux pas que tu te déplaces dans des voitures dernier modèle, je ne veux pas que tu t'achètes de villas, je veux que tu adoptes un profil bas, discret, tranquille. Si tu enfreins ça, t'es un homme mort. T’es prévenu, pas d'embrouille."

[A moi :] C'étaient les accords... [...] Ça se passait comme ça :

- "Chef ?

- "Oui.

25. La «place» est l'espace géopolitique (une ville et ses environs le plus souvent) sur lequel un trafiquant et son organisation exercent le droit exclusif (acheté au pouvoir exécutif à l'époque décrite par Don Silvio) de contrôle sur le trafic, la distribution de drogues et leur transit par ce territoire. 
- "Juan Perez est là qui veut vous parler.

- “C’est qui celui-là ?

- “C'est l'capo de Sonora.

- “Fais-le entrer ! [Il entre.] Qu'est-ce qu'y a ? Que veux-tu ?

- [Changeant de ton, prenant l'accent d'un cultivateur du Nord] “Eh bien, c'est pour un coup de main chef. J'en peux plus des soldats et j'ai la police fédérale sur le dos, je leur donne tant d'argent mais y'a pas de fin à leur racket, total que j'm'en sors pas... Donnezmoi un coup de main.

- [Dans son rôle du fonctionnaire de Gobernación] "OK. Combien tu paies?

- [Le cultivateur, d'un ton plaintif] "J'paye un million de dollars par mois pour ma protection.

- [Le fonctionnaire] "OK. Et qui tu paies ?

_ "Ben lui et lui. Et lui aussi.

- “OK. Et tu veux faire quoi ?

- “Bon, moi, le fric, je m'en fiche, quitte à les payer le double pourvu qu'ils respectent nos accords : celui-ci j'le paie et il tient pas sa parole, ils m'ont saisi mes cargaisons sur la nationale, mes avions ont été immobilisés à l'aéroport et c'est un désastre et à moins de tuer le colonel, le chef de la police fédérale ou celui de la police de l'Etat, on fait quoi ?"

- [S'adressant à moi] C'est là qu'entre - est entré - en scène Gobernación, pour commencer à mettre de l'ordre. On contacte le colonel en charge de la zone, le chef de la police fédérale de la zone, le chef de la police d'Etat de la zone, le chef de la police municipale... et on les fait asseoir !

- "J'suis la CIA mexicaine et pour des raisons de sécurité, ce type dorénavant c'est moi qui m’en charge, vous me le laissez... vous en mêlez pas !” [Il rit] [...]

- [SG] Il leur dit quoi ?

- [DS] De plus l'inquiéter. Qu'à partir de maintenant...

- [SG] ... il est protégé...

- [DS] Il est protégé par moi ! On n'avait qu'une parole... ça valait pour un ordre, tu comprends ? Historiquement, le gouvernement fédéral gérait le narcotrafic, à l'exception de certains gouverneurs qui s'y immisçaient de plain-pied, à leurs risques et périls. [...] [Résumant la configuration des rapports gouvernement/narcos jusqu'à la deuxième moitié des années 1980] Donc, ce n'est pas que le gouvernement dirigeait : il coordonnait, il arrangeait... Les disputes, les procès, les zones, les territoires... Le gouvernement ordonnait, maintenait une paix. Il organisait, pas directement. La fonction du gouvernement était de protéger le narcotrafic... C'était la protection. Quel genre de protection ? Les champs, qu'on ne les saisisse pas. Le transport des drogues, qu'on ne les confisque pas. [...]» 
Dans les années 1980, les narcotrafiquants étaient encore dans une position claire de subordination au pouvoir politique ${ }^{26}$, c'est ce que nous dit le récit de Don Silvio ${ }^{27}$. On y entend s'élever la voix du cultivateur qui veut mener à bien son travail (de production et d'exportation de marijuana) et, pour ce faire, paie un tribut à des agents du gouvernement. Lui répond un haut représentant du gouvernement fédéral ou de l'Etat de Chihuahua, non identifié dans un premier temps, puis, clairement, un fonctionnaire de la Secretaría de Gobernación (Don Silvio lui-même, peut-être). Ces employés du gouvernement, en échange de leur quote-part, garantissaient un environnement politique et social stable, donc propice aux affaires.

Ensemble, les trafiquants à temps plein et les «facilitateurs » des trafiquants - des individus issus de toutes les classes sociales 28 - étaient les maillons accrédités d'une chaîne de coopération au narcotrafic. La frontière était l'instrument de leur commune ambition de maximiser les profits à tirer du commerce de la drogue. Car une fois la frontière franchie, selon les termes de Don Silvio, le narcotrafic «cesse d'être un jeu d'enfants pour se convertir en grand négoce »- les sommes en jeu sont décuplées. Aujourd'hui encore, comme le dit la maxime, "qui contrôle la frontière, contrôle le trafic»; les organisations qui ne la contrôlent pas doivent négocier et payer des droits de passage à qui domine la zone. La frontière est ainsi un enjeu de dispute entre organisations criminelles dans la période récente. Mais, à l'échelle des transporteurs de cargaisons de stupéfiants, tout à leur préoccupation d'éluder les contrôles sur la route du Nord, la frontière est aussi le point de mire. De même pour des petits trafiquants qui s'y rendent en quête de travail, elle est un objet de convoitise et d'espérance.

26. Luis Astorga a bien montré la relation de subordination des trafiquants mexicains à l'Etat sous le règne du Partido Revolucionario Institucional, voir notamment El siglo de las drogas, op. cit.

27. Dont le contenu vient en appui d'autres récits recueillis sur le terrain, auprès d'autres acteurs, qui expriment autant de points de vue sur la période en question. Alban Bensa (« Père de Pwädé. Retour sur une ethnologie au long cours », Bensa A., Fassin D. (dirs.), Les Politiques de l'enquête. Eprenves ethnographiques, Paris, La Découverte, 2008) souligne l'importance de "penser [1']enquête comme une histoire au sein de laquelle [nos] interlocuteurs jouent leurs propres intérêts ». De fait, si Don Silvio a observé « de l'intérieur » ces rapports très étroits entre trafiquants et gouvernants, ses mots gagnent à être replacés dans le contexte spécifique d'interlocution : face à lui qui se posait en historien des rapports entre Etat et narcotrafic, j'occupais la position de l'étudiante et je lui fournissais l'occasion de replonger dans ses souvenirs et de revivre un passé où il occupait une position d'autorité (il ne travaille plus pour le gouvernement fédéral), c'était donc l'occasion de se couler à nouveau dans cette position et aussi de faire revivre une époque - dans l'histoire du narcotrafic - souvent racontée avec émotion par mes interlocuteurs. Le savoir que Don Silvio me livre reste par ailleurs à un niveau de généralité - d'une part nous ne nous connaissions pas assez pour qu'il en soit autrement, d'autre part il écrivait un roman sur cette période et il était soucieux de ne pas « dire tout » ce qu'il avait prévu de raconter sous la forme d'une fiction. Concernant les liens qui unissent Don Silvio et Roberto mon « passeur ", par-delà leur amitié, des éléments précieux me manquent pour comprendre la décision du premier de me parler.

28 . Outre ceux ici mentionnés, des économistes, des financiers, des administrateurs, des comptables, des avocats, des ingénieurs, des membres du département de sécurité (tueurs à gage, gardes du corps, protecteurs des convois, gardiens d'entrepôts), des distributeurs, etc. Sur cette chaîne de coopération, Guez S., op. cit. 
Leurs déplacements sur la carte du Mexique, à l'instar des négociations dans les capitales, entre les « conseillers » desdits cartels et leurs protecteurs au sein de l'Etat mexicain dans la période évoquée par Don Silvio, nous donnent à penser la frontière dans sa relation avec l'intérieur du pays. La frontière Ciudad Juárez - El Paso ne cesse pour autant à aucun moment d'être un lieu; Don Silvio a bien su transmuer la parfaite connaissance qu'il en avait en carrière. Sa discrétion et son choix de fermer les yeux lui ont valu, en haut lieu, des amitiés. La confiance qu'il s'assurait par son silence tacite sur des pratiques d'entente illégales, le savoir acquis sur ces ententes, il les traduira, une fois dissous le système DFS/DGIPS, en possibilité d'exercer d'autres fonctions dans l'administration politique de la frontière. Une infinité d'autres circulations relient cette dernière à l'intérieur états-unien, comme l'histoire ci-après de Ricardo le suggère.

\section{Ricardo, de passage à El Paso}

Pour l'élite des trafiquants comme pour leurs petites mains, la frontière est un défi, le point de passage obligé, but du jeu pour les premiers, va-tout angoissant des seconds. Du point de vue de Ricardo, qui avait pris goût à l'argent, elle était la solution.

Ricardo, 32 ans, trafiquant de mère en fils, m'a été présenté par une amie commune, Caramela, la femme qui fut mon " passeur ». Il faisait une halte à El Paso ce jour-là. Il devait prendre un car pour Denver où il récupérerait près d'un million de dollars en paiement d'une cargaison de cocaïne, qu'il rapatrierait au Mexique camouflé dans la voiture qui l'attendait dans le Colorado. A qui remettrait-il cette somme ? Pour qui travaillait-il ? Autant de questions qu'on ne pose pas. Disons, sans risque de se tromper, que Ricardo travaillait au sein d'une des myriades de cellules qui composaient l'organisation régnant alors sur le territoire chihuahuense, celle de la famille des Carrillo Fuentes (dite "Cartel de Juárez »). Il arrivait de Chihuahua - capitale d'où Caramela avait, elle, émigré quatre ans auparavant avec une idée en tête : son fils handicapé, né aux Etats-Unis, aurait accès dans ce pays aux meilleurs soins de santé. Un nouveau départ pour Caramela, ancien mannequin, négociante en immobilier pendant 15 ans à Chihuahua. Les cours d'anglais qu'elle suivait à l'université publique d'El Paso étaient financés par une bourse d'étude. Ses faibles revenus lui donnaient droit à d'autres aides sociales, au montant dérisoire et continuellement rognées, qui faisaient dire à des Mexicains rêveurs que les Américains qui les percevaient étaient des «güeros felices " : des «blonds heureux » qui ne savaient pas ce que c'était que travailler. Mais Caramela avait un petit boulot déclaré et un autre qui ne l'était pas ; cette situation qui ressemblait à s'y méprendre à un déclassement social n'était pas précisément un choix. Outre le souci du bien-être de son fils, il y avait aussi à l'origine de leur installation à El Paso une volonté de Caramela de «clore des chapitres", comme elle disait. A Chihuahua, elle avait été l'égérie de plusieurs trafiquants 
et connu la vie de château, les années de fêtes et d'insouciance. L'emballement de la violence liée au trafic avait fini de recouvrir d'oubli le temps où les gens de la «bonne» société s'acoquinaient avec des trafiquants de drogue. Caramela, elle, n'oubliait rien : beaucoup de ses amis étaient morts, d'autres amis avaient "disparu", leur corps n'avait jamais été retrouvé; d'autres encore avaient développé une dépendance à la base ${ }^{29}$. Ses proches voyaient ses fréquentations d'un mauvais œil. Mais Caramela était fidèle en amitié et Ricardo était toujours le bienvenu chez elle à El Paso. C'était un petit gabarit, avec l'air assuré et amusé, un œil de renard. Il était sec mais une grande douceur émanait de lui quand il s'adressait à elle. Entre eux aucune équivoque ne subsistait, mais de l'amitié fraternelle, qui ne méconnait pas l'autre, construite au long cours.

«Sacré Ricardo... il est né à Naranjo, Sinaloa!» disait-elle en riant, comme si ça expliquait tout. Le Sinaloa est, en effet, le berceau du trafic, la terre d'origine des grandes lignées de trafiquants ${ }^{30}$. Dans les environs montagneux de Naranjo, on croise des enfants, des adolescents et leurs grands-pères dans des villages où la génération des pères n'est plus, décimée dans les années 1990 par les règlements de compte. C'est pour échapper à ce sort que Ricardo et sa famille avaient émigré dans l'Etat limitrophe de Chihuahua, parcouru, comme celui de Sinaloa, par la Sierra Madre occidentale, région de culture de cannabis et de pavot depuis au moins trois générations.

«La moitié de la famille se consacre à ça, imagine : c'est tout ce qu'ils savent faire. [...] Le père est le seul à pas être mouillé, le pauvre homme... Il est ingénieur en hydraulique et sa femme est la plus déchaînée de tous ! Jusque-là [main au front] dans le narco ! »

Quand Caramela m'a présenté Ricardo, sa mère purgeait une peine - pas la première - de cinq ans dans une prison de Seattle. Au poste-frontière, les inspecteurs avaient découvert sur elle $6 \mathrm{~kg}$ de cocaïne. Derrière les barreaux, une conduite irréprochable : elle enseignait l'espagnol et serait transférée bientôt dans la prison de Chihuahua-capitale bien que ne le souhaitant pas car, làbas, disait Ricardo, tout se monnaye. A Chihuahua, Ricardo venait de fêter l'ouverture de son restaurant de fruits de mer et spécialités sinaloenses. Il l'avait inscrit au registre du commerce au nom de son père car, lors de son dernier séjour à «l'uni » (la fac, c'est-à-dire sous les verrous), son épouse avait croqué l'argent de la drogue, toutes leurs économies. Il marchait sur les pas de sa mère depuis longtemps et trompait bien son monde au moment où lui et

29. Forme pure de l'alcaloïde cocaïne, fumable, la base est l'agglomérat pâteux qu'on obtient en « cuisinant » le chlorhydrate de cocaïne par adjonction de bicarbonate de soude ou d'ammoniaque.

30. Voir Astorga L., "Géopolitique des drogues au Mexique. L’hégémonie des Sinaloans sur le trafic des drogues illicites ", Hérodote, $\mathrm{n}^{\circ} 112,1^{\mathrm{er}}$ trimestre 2004 ; Astorga L., El siglo de las drogas, op. cit. 
Caramela s'étaient connus. Il travaillait alors pour un grand magasin de fournitures de bureau mais c'était une couverture. En réalité, il était dealer-livreur. Il en imposait dans son costume avec son attaché-case qui ne contenait pas que les bons de commande - comme longtemps Caramela l'avait cru - mais aussi la consommation personnelle en stupéfiants de son patron à elle. Elle aussi était devenue sa cliente, leur amitié était née ainsi. Elle lui a sauvé la mise deux, trois fois à El Paso. Ainsi, un an auparavant, racontait-elle : "Ceux qui avaient passé la coca l'avaient jetée sans précaution ni soin dans la voiture... ", charge à Ricardo et à son acolyte de l'empaqueter dans les règles de l'art. Les deux hommes s'étaient installés dans l'hôtel où Caramela avait un temps résidé à son arrivée, pas loin du centre-ville, et «ils [avaient] acheté le matos nécessaire : la thermoscelleuse pour extraire l'air des sacs en plastique dans lesquels ils [avaient] mis la cocaïne... ", sacs ensuite recouverts de feuilles d'aluminium puis de papier carbone et enfin, une nuit, dissimulés dans le radiateur de la troca (la camionnette). Caramela leur avait permis de la garer à l'emplacement de sa propre voiture sur son parking. Elle leur amenait des petits plats à l'hôtel. En partant, ils lui avaient fait cadeau d'une bolsota (un gros sac) de cocaïne en plus du matériel («ils en avaient eu pour 250 dollars de matériel»). Toutes actions accomplies à El Paso, gestes simples et indispensables à l'effectuation du narcotrafic. Des services rendus à un trafiquant par amitié, un coup de main parfois rémunéré venant en appoint d'un revenu légal. Leur prestataire, ici Caramela, devenait un maillon intermittent de la chaîne de coopération au trafic des stupéfiants, sans pour autant devenir ni se penser à aucun moment comme une trafiquante.

Sur une suggestion de Caramela, je l'avais emmené chez Walgreens acheter une couverture et un oreiller de voyage pour les onze heures de route qui l'attendaient. Son visage impassible m'avait frappée : pas une ride d'expression. Ses yeux fixés dans les miens, ses « conseils d'ami $»$ comme il disait :

«Poser des questions, c'est dangereux, ne te fie à personne, qui sait si la personne à qui tu es en train de t'adresser est un assassin, un tueur professionnel ? à El Paso, des tas de gens se cachent, recherchés au Mexique. Ils craignent pour leur vie à Juárez et se réfugient ici... Des voitures passent la frontière "pleines" tous les jours, c'est un commerce florissant et un va-et-vient incessant. A Juárez, des gens travaillent avec moi, ils se tiennent prêts pour moi, et d'eux non plus, il n'est pas possible de dire en les voyant qu'ils en vivent. Je ne connais ni leur nom ni leur téléphone, on a tous des surnoms. Qui est le chef ? Je n'en sais rien. J'ai un interlocuteur, il en a un au-dessus de lui et ainsi de suite... »

Il m'avait confié l'avoir échappé belle six mois plus tôt, en fait, selon Caramela il s'en remettait à peine : lui et le type qui conduisait s'étaient fait prendre au Pont international des Amériques, ils transportaient de la cocaïne, 
«on nous a balancés» (《nos pusieron el dedo»), Ricardo avait été interrogé pendant près de quinze heures et son associé avait maintenu que Ricardo n'était au courant de rien, il n'avait pas flanché. Il est toujours en prison. Depuis, Ricardo franchissait à pied et les mains dans les poches la frontière dans le sens Mexique/Etats-Unis mais il n'en avait pas moins droit à l'interrogatoire, qui avait duré cette fois-ci cinq heures. Il ne passait plus de drogue. Il avait bien essayé, un mois après l'interpellation : une cargaison de marijuana. Il n'avait pas réussi. A son chef il avait dit qu'on lui avait retiré son permis mais à Caramela il avait avoué en pleurs avoir la trouille. Il avait rebroussé chemin et un autre s'était chargé du transport. Il ne franchissait plus la ligne de démarcation qu'avec l'argent des transactions : au poste-frontière, en direction du Mexique " on te demande rien ».

C'était notre première rencontre. Sept mois s'écoulèrent avant la dernière, au parloir, il était en détention préventive dans cet immense cube de béton sans fenêtres qui fait face au tribunal fédéral d'El Paso. Entre-temps, il s'était senti pousser des ailes mais aux yeux de Caramela il se les était brûlées.

Il avait pris de l'assurance et ses contacts l'avaient conduit à Dallas, puis de nouveau au Colorado où il avait décroché un marché. Nul petit entrepreneur ne pouvant trafiquer de la cocaïne à son compte, il s'agissait de marijuana. Jusque-là, Caramela ne trouvait rien à redire - c'était ce que son ami savait et voulait faire, il comptait sur elle, et peut-être en espérait-elle un juste retour mais, quel souci ! elle n'en dormait pas la nuit s'il oubliait de donner de ses nouvelles; et elle estimait son dévouement pour les siens et sa force au travail : en plus de tenir son restaurant, il revendait du matériel hi-fi mis en gage, vendait des voitures d'occasion, plus le trafic.

Mais il commettait des erreurs. La première, selon elle : s'associer avec un homme de Chihuahua, comme lui, qui ne lui faisait pas confiance. De suite il y eut des ennuis. Deux véhicules devaient traverser la frontière, et l'homme âgé qui devait conduire le premier prit peur et se dédit devant l'état de délabrement du «mueble» («babut», ici une camionnette). Pour ne rien arranger, la citoyenne américaine qui d'ordinaire "passait » également des cargaisons semblait s'être volatilisée. Ricardo crut donc bon d'acheminer jusqu’à Denver le deuxième véhicule arrivé, lui, sans encombre à El Paso : de retour du Colorado il trouverait un passeur pour le premier. L'associé dépêcha plutôt un de ses jeunes depuis Chihuahua, qui récupéra à Ciudad Juárez la camionnette et passa au travers des contrôles au poste-frontière: le jeune avait un parent à Sunland Park au Nouveau-Mexique. Mais Ricardo s'opposa à ce qu'il y laisse la troca plusieurs jours : il n'avait pas plus confiance en son associé que ce dernier en lui, et il connaissait un lieu sûr en la résidence de Caramela.

Seconde erreur, qu'elle ne lui pardonna pas : cédant à la pression, Ricardo avait communiqué son adresse à l'associé. Exposant Caramela et son fils à un 
danger qu'elle lui en voulait de n'avoir pas apprécié (en cas de vol de la camionnette, l'associé pourrait l'en estimer responsable et, qui sait, les faire payer de leur vie). On partait elle et moi le lendemain en voyage, son fils serait gardé par sa nounou. A mesure que nous nous étions éloignés de la frontière, et de jour en jour, son angoisse grandissait, de même la fureur que nourrissait son analyse de la situation. Au retour à El Paso, au terme d'un enchaînement de développements qui l'avaient conduite, entre autres à devoir trouver une planque pour la camionnette dans l'attente de Ricardo, retenu au Colorado, les deux amis depuis toujours, se brouillèrent.

On n'entendit plus parler de Ricardo jusqu'à ce que, deux mois plus tard à Chihuahua, un proche de Caramela le lui apprenne : au checkpoint de la Border Patrol sur l'autoroute 62-80 à Desert Haven, Texas, à l'Est d'El Paso, Ricardo, ainsi qu'une femme qui voyageait à ses côtés et un enfant sur le siège arrière, avaient été interpellés. De Chihuahua, pas le moins du monde étonnée, Caramela égrenait par téléphone les maigres faits dont ils disposaient là-bas, qui venaient de faire $350 \mathrm{~km}$ en sens inverse : Ricardo avait appelé de la prison, à El Paso, et il n'avait donc pas pu beaucoup parler; la famille de la femme sur le siège du passager avait eu d'autres éléments. Caramela me les livrait d'une manière clinique, celle d'une personne en proie à des pensées graves - un parent aimé venait de décéder, raison de sa présence à Chihuahua. Elle en voulait toujours à Ricardo mais leurs liens affectifs se révélaient forts, ils avaient survécu aux motifs de leur rupture. Elle espérait de moi sur place à El Paso que je puisse en apprendre davantage, pour leurs proches à tous là-bas à Chihuahua.

Au parloir, face à moi, Ricardo était confiant, pour $30 \mathrm{~kg}$ il ne serait pas condamné à une peine trop longue. D'autant qu'il n'y avait pas récidive : lors de son interpellation précédente, il avait donné un faux nom. Il m'assura que la femme qui l'accompagnait, dans le rôle de l'épouse, était sans aucun doute libre à cette heure parce qu'il n'avait eu de cesse, auprès de ses interrogateurs, de clamer son ignorance. Dans le « communiqué des faits » rédigé par l'agent de la Drug Enforcement Administration (DEA), adjoint à la plainte légale déposée contre lui au lendemain de leur arrestation dont j'avais plus tard pu obtenir copie, il était au contraire écrit que « [les deux] ont admis qu' [elle] recevrait 1000 dollars pour son assistance ». Quelqu'un mentait - l'agent de la DEA ou Ricardo.

Quel qu'il soit, Caramela se dit lasse des choix de Ricardo qui, une fois encore, s'était arrogé le droit de mettre en danger un enfant pour tromper les soupçons. Des circonstances exceptionnelles - l'incarcération de Ricardo avaient relégué le ressentiment de Caramela à l'arrière-plan. Ses éventuelles récriminations se dissipaient dans cette situation d'urgence et l'intérêt de leur communauté d'amis primait sur le sien propre. Mais le détail de ce qui s'était joué avait ravivé le souvenir de la trahison de Ricardo deux mois auparavant et attisait sa déception. Il soulignait la divergence de leurs morales, de leurs codes respectifs quant au possible et au juste. 


\section{La frontière en son au-delà}

Que j'écoute leurs récits, que j'imagine mes interlocuteurs dans leurs cheminements biographiques et je suis transportée en d'autres temps et lieux. Leurs parcours, leurs histoires dessinent autant de fils invisibles sur toute la géographie nationale du Mexique et des Etats-Unis et ces mouvements de noria traversent la frontière, comme les stupéfiants et l'argent de leur trafic dont il a été ici question. La frontière, ces personnes l'établissent aussi entre les choix qu'ils s'autorisent ou non. Le tracé de cette frontière-là est mouvant : il s'apparente plutôt à une gradation floue entre ce qui se fait et ce qui ne se fait pas, indissociable du contexte sociohistorique au moment dit. Les stupéfiants en route pour les marchés du Nord portent trace de tous ces franchissements, et dans une infinité de lieux et de pratiques à la frontière, les traces de leur commerce, et de son interdiction, sont présentes.

Pour ne parler que de la sphère économique, Don Silvio estime que «sur dix dollars introduits dans l'économie de Juárez, trois viennent du narcotrafic ». Ses traces sont également observables en maints espaces sociaux à El Paso - on peut par exemple légitimement s'interroger sur la hausse du prix de l'immobilier dans le troisième comté le plus pauvre du pays ${ }^{31}$. Notable aussi, le semis de signes qu'y imprime la politique de Washington en matière de répression des flux de stupéfiants. Elle s'était intensifiée dans les années 1980, sous Ronald Reagan, puis ce fut l'escalade dans les années 1990. D'extraordinaires moyens sont mis en œuvre, côté états-unien : quelque cinquante agences fédérales ont autorité pour procéder aux arrestations de trafiquants, sans compter "les milliers d'agences au niveau de l'Etat et des municipalités qui effectuent des saisies » dans ce territoire frontalier officiellement baptisé " aire de trafic de drogue de haute intensité » ${ }^{32}$. Le produit de la vente des biens confisqués aux trafiquants (véhicules, maisons-entrepôts) fait l'objet d'âpres partages entre agences. La "guerre » livrée à la drogue - déclarée en 1971 par Richard Nixon dans la perspective d'épargner aux Républicains une défaite dans les élections au Congrès et d'asseoir son propre pouvoir ${ }^{33}$ - est aujourd'hui localement une source majeure d'emplois publics et privés.

Ainsi, en face du El Paso County Detention Facility, lorsque Ricardo y était incarcéré, s'étendait sur un pâté de maisons l'immense chantier du nouveau tribunal fédéral; ses dimensions seraient à la mesure du défi posé par les réseaux d'acteurs qui jouent sur les politiques de prohibition.

31. Hausse lorsque j'étais sur le terrain. Voir Guevara K., «Es condado de EP tercero más pobre ", El Diario de El Paso, 13 novembre 2006.

32. Statistiques de la Drug Enforcement Administration, entretien avec David Monnette, juin 2006.

33. Sur la "guerre contre la drogue ", voir Epstein J., Agency of Fear. Opiates and Political Power in America, New York, Putnam, 1977, http://edwardjayepstein.com/agency/prologue.htm ; Baum D., Smoke and Mirrors: The War on Drugs and the Politics of Failure, Boston, Little Brown \& Co., 1996. 